\title{
Designing to Support Communication on the Move
}

\author{
Jacqueline Brodie \\ Department of Information Systems and Computing \\ Brunel University \\ Uxbridge, UB8 3PH UK \\ +4418952740000 \\ Jacqueline.Brodie@brunel.ac.uk
}

\begin{abstract}
We investigated what mobile workers do when they are mobile to achieve their communication goals, using contextual interviews and ethnographically inspired observations in a variety of settings. Implications for the design of mobile technology were extracted from the raw data collected in the fieldwork using such novel design techniques as 'Day in the Life' vignettes, affinity diagrams, and consolidated artefact models from Contextual Design. Our findings are being using to generate software prototypes for supporting mobile activities.
\end{abstract}

\section{Keywords}

Mobile work, fieldwork, Contextual Design, prototyping

\section{INTRODUCTION}

The nature of work and the workplace is changing with the arrival of hot desking; tele-working and increasing mobile work. Likewise, a variety of new and emerging technologies in the marketplace have ushered in increased communication capability and connectivity for mobile users. Future technology will no doubt seek to harness such innovations as Wireless LAN, Bluetooth, GPS, 3G and GPRS. However, despite such technological developments the key problem for designers and developers of mobile technology is how to combine such a myriad of new protocols and new technologies into applications and hardware that truly support the dynamic and flexible communication needs of the mobile worker population.

There is no definitive definition of 'mobile work'. However, for the purposes of requirements capturing we can see mobile work activities as involving "working at multiple (but stationary) locations, walking around a central location, travelling between locations, working in hotel rooms, on moving vehicles and in remote meeting rooms" [1]. Literature review reveals no-one method to capture design requirements for mobile technology. However, previous ethnographic studies of technology in the workplace, with their emphasis on the user in context and the deployment of user-centred interactive design method-

Copyright is held by the author/owner(s).

CHI 2003, April 5-10, 2003, Ft. Lauderdale, Florida, USA.

ACM 1-58113-630-7/03/0004. ologies seems a fruitful direction to explore $[2,3,4]$. These entail a move away from the laboratory based approaches of requirement capturing and into the arena of methods that assign the user a design role that is more participatory $[5,6]$. Past studies have identified problems that result from not making use of user-centred design methodologies when creating mobile systems. Such problems as duplication of users' time and effort through workarounds; repetition of information gathering activities and redundant technology have been well documented [4]. Given the already challenging nature of mobile work for users of mobile technology - with a context that is constantly changing as people interact and re-orient themselves to their environments [7] - our study seeks first to understand the work that mobile workers do, and then design software that complements it.

\section{METHODS}

In order to identify and support the diverse communication needs of mobile workers, we explored and combined existing research methods for data collection. This was to allow the identification of common issues across a variety of mobile technology users - be they mobile white-collar managers or blue-collar plumbers [8]. The methods used to interpret our field data were similarly diverse [5]. This was intentional, so as to allow a comprehensive grasp of the needs of the varied user population we are researching and also to aid more appropriate representation of coordination on the move. The research methods used drew on ethnographic techniques for data gathering, as well as HCI methods, like prototyping, that allow real world iterative evaluation of the use of mobile communication technology.

Fifteen contextual interviews with photographs detailing the artefacts used by our participants were undertaken. This work was supplemented by ethnographically inspired observations on trains and at airports of mobile workers in action. In addition, detailed analysis of the interview transcripts has been carried out to provide not only a comprehensive background understanding of mobile device users, but to ensure that the demanding nature of mobile communication is fully documented, and the physical, social, technological and organisational constraints that any mobile application will function under are recorded [8]. Some consolidated artefact models were created and Affinity diagramming [5] was carried out to help in the identification of common themes across our diverse user 
groups. These themes are being used to inform and guide our prototype development. The understandings gained from the fieldwork have been fed into the construction of rich composite user vignettes (Day in the Life Stories) that portray the current working practices of mobile workers. These are currently being used to inspire and justify prototype designs of mobile communication technology in the development phase of the research.

\section{FINDINGS AND DESIGN IMPLICATIONS}

The initial stages of the research gathered insights into how mobile workers operate; the problems they face; the strategies and techniques that they use to communicate with mobile or static colleagues in the workplace [8]. Below we discuss key themes emerging from the data and their corresponding implications for design:

1. Incoming information on one medium tends to stay on that medium, e.g. paper to paper, email to email and phone to phone. Only occasionally do cross-media transitions occur - for mainly record keeping - since such transitions are time consuming and cognitively expensive. Designers of mobile devices can support such a finding by providing mobile tools that facilitate and simplify cross-media transitions (or design so that cross-media transitions are not required).

2. There is an expectation of anytime, anywhere access to email/voice mail and other information sent by colleagues and clients. However, this is not always matched by the mobile worker's ability to communicate in these timeframes, leading to confusion and frustration. A possible design solution for this problem would be an 'awareness' tool that informs colleagues that a user is busy with other activities and unable to respond at the current time. While awareness tools (e.g. IM) are currently available, they are not explicitly suited to mobile work and mobile devices.

3. Mobile workers often work with other members of their community of practice (or community of knowledge) while on the move. However, current digital technology often lacks adaptability (for example, 'orientation' flexibility) and this decreases the shareability of such resources. This demonstrates a need to fully integrate such support into future cellular/wireless technologies in order to facilitate collaborative mobile activities.

4. Pre-planning before mobile work was often necessitated by the current form factor of communications technology. Mobile workers sought out flat surfaces to work on, e.g. tables in restaurants and bars in airports. This often led to breakdowns with users unable to work just anywhere. Such findings imply a greater focus is needed from technological designers on the context of use that any technology will be used in. It also suggests two solution paths: roomware to support mobile technology, such as electronic tables, or an emphasis on making mobile technology more intrinsically mobile.

\section{FUTURE WORK}

This paper has outlined research into requirements capturing for the design of technology to support the communicative needs of mobile workers and identified how the data collected in the research has been fed into the design process for new mobile software. Our research findings have shown that a key future challenge for developers and designers is how to integrate wireless technology for practical use in mobile devices for a diverse user population. Currently, we are selecting mobile worker activities that are to be augmented by technology and the potential advantages that such additional technology could provide for the users and their communities. Our first prototype, inspired by our findings, is an 'awareness' tool that allows mobile workers the opportunity to have additional text notification of their mobile phone voicemails, via their phone. This software allows organisation of the order they will listen to the calls and provides a permanent archive of all voice calls received. Iterative prototyping development will be carried out on this tool and user evaluation studies, using typical users of mobile devices, are also planned. This will motivate future redesign of the technology and offer insight into the potential effects the technology might have on the work itself.

\section{ACKNOWLEDGMENTS}

Thanks to my supervisor Mark Perry for his advice and support.

\section{REFERENCES}

[1] Perry, M. et al. Dealing with Mobility: Understanding Access Anytime, Anywhere. ACM Transactions on Computer-Human Interaction, vol. 8,4 (2001), 323 - 347.

[2] Anderson, R.J. Representations and Requirements: The Value of Ethnography in System Design. Human-Computer Interaction, Vol. 9,2 (1994), 151- 182.

[3] Bellotti, V. \& Bly, S. Walking Away from the Desktop Computer: Distributed Collaboration and Mobility in a Product Design Team. In Proceedings CSCW '96 (Boston, Massachusetts, September, 1996). ACM Press, 209-218.

[4] Luff, P. \& Heath, C. Mobility in Collaboration. In Proceedings of CSCW'98 (Seattle, Washington, November, 1998). ACM Press, 305-314.

[5] Beyer, H. \& Holtzblatt K. Contextual Design. San Francisco. Morgan Kaufman (1997).

[6] Vanaanen-Vainio-Mattila, K. \& Ruuska, S. User Needs for Mobile Communication Devices. First Workshop on HCI for Mobile Devices, (Glasgow, Scotland, May 1998).

[7] Kristoffersen, S. \& Ljungberg, F. Making Place to Make IT Work: Empirical Explorations of HCI for Mobile CSCW. In Proceedings of Group'99 (Phoenix Arizona, November, 1999). ACM Press, 276-285.

[8] Brodie, J. \& Perry, M. Designing for "Blue-Collar" Work. Mobility, Collaboration and Information Use. $A C M$ Siggroup Bulletin, 22, 3 ( 2001), 22 - 27. 